\title{
Insegurança alimentar das famílias residentes em municípios do interior do estado da Paraíba, Brasil ${ }^{1}$
}

\section{Household food insecurity in municipalities of the Paraíba State, Brazil}

Rodrigo Pinheiro de Toledo VIANNA 2

Ana Maria SEGALL-CORRÊA ${ }^{3}$

RE S U M O

\section{Objetivo}

Identificar a prevalência de segurança alimentar e dos diferentes graus de insegurança alimentar entre famílias residentes em 14 municípios da Paraíba e a relacionar com o perfil social, demográfico e econômico destas famílias.

\section{Métodos}

Realizou-se inquérito populacional no qual 4533 famílias foram entrevistadas. Utilizou-se questionário para avaliar as características sócio-demográficas, juntamente com a Escala Brasileira de Insegurança Alimentar. Foram calculadas medidas de prevalência e verificada a associação entre as variáveis do estudo pelo teste qui-quadrado. Ajustou-se um modelo de regressão logística para examinar a associação dos fatores de risco à segurança e à insegurança alimentar.

\section{Resultados}

Observou-se prevalência de $11,3 \%$ de insegurança alimentar grave, 17,6\% de insegurança moderada, 23,6\% de insegurança leve; $47,5 \%$ da população foi classificada em situação de segurança alimentar. As áreas rurais apresentaram pior situação. 0 principal motivo referido para insegurança alimentar foi a falta de dinheiro para aquisição de comida. A regressão logística final foi composta por três variáveis: baixa renda familiar per capita ( $R \$ 25,00$ versus $R \$ 300,00$, Odds Ratio $=19,10$ ), moradia precária (Odds Ratio $=1,98$ ) e falta de água permanente (Odds Ratio $=1,38$ ). As famílias do menor estrato de renda apresentaram menor prevalência de insegurança alimentar grave, quando incluídas em programas sociais.

\footnotetext{
1 Projeto de pesquisa "Avaliação da insegurança alimentar de famílias residentes no estado da Paraíba". Apoio financeiro: Ministério da Ciência e Tecnologia, Ministério do Desenvolvimento Social e Combate à Fome e Conselho Nacional de Desenvolvimento Científico e Tecnológico, a partir do Edital CT-Agronegócio 01/2003 (processo CN Pq № 503359/ 2003-3).

2 Universidade Federal da Paraíba, Centro de Ciências da Saúde, Departamento de Nutrição. Campus Universitário I, 58059-900, João Pessoa, PB, Brasil. Correspondência para/Correspondence to: R.P.T. VIANNA. E-mail: <vianna@ccs.ufpb.br>. 3 Universidade Estadual de Campinas, Faculdade deCiências M édicas, Departamento de M edicina Preventiva eSocial. Campinas, SP, Brasil.
} 


\section{Conclusão}

A prevalência de insegurança alimentar foi elevada. 0 instrumento utilizado demonstrou ser uma ferramenta importante de avaliação da situação de segurança alimentar e útil para o monitoramento de políticas públicas, como é o caso dos programas sociais que integram a estratégia Fome Zero do Governo Federal.

Termos de indexação: Estudos transversais. Epidemiologia. Segurança alimentar e nutricional.

\section{A B S T R A C T}

\section{Objective}

The objective of this study was to identify the prevalence of household food security in 14 municipalities of Paraiba State and examine its association with the social, demographic and economic profile of these families.

\section{Methods}

A cross-sectional study comprising 4,533 families was performed. A questionnaire was used to assess the sociodemographic characteristics together with the Brazilian Food Insecurity Scale. The prevalences were calculated and the association between the studied variables was verified by the chi-square test. A logistic regression model was adjusted to identify risk factors associated with food security and insecurity.

\section{Results}

The prevalence of food insecurity was $11.3 \%$ for severe, $17.6 \%$ for moderate and $23.6 \%$ for mild. Food security was found in $47.5 \%$ of the families. The situation in rural areas was worse than in urban areas. Insufficient money to buy food was the main reason reported for the food insecurity status. The final logistic regression consisted of three variables: low per capita family income (US $\$ 12.00$ vs. US $\$ 150.00$, Odds Ratio $=19.10$ ), poor household conditions (Odds Ratio $=1.98$ ) and permanent absence of water supply (Odds Ratio=1.38). A lower prevalence of severe food insecurity was found among families with per capita monthly income up to US\$12.00 that were included in social programs.

\section{Conclusion}

The prevalence of food insecurity was high. The methodology used proved to be an important assessment tool of food security status and useful to monitor public policies such as the social programs that are part of the Fome Zero (Zero Hunger) strategy of the Federal Government.

Indexing terms: Cross-sectional studies. Epidemiology. Food security.

\section{N T R O D U C Ã O}

Segundo a classificação do Programa das Nações Unidas para o Desenvolvimento (PNUD), - Estado da Paraíba está entre as regiões consideradas de médio desenvolvimento humano (Índice de Desenvolvimento Humano - IDH entre 0,5 e 0,8). Em relação aos outros estados do Brasil, a Paraíba apresenta-se em situação desfavorável, ocupando a 24a posição (Índice de Desenvolvimento Humano Municipal - IDHM $=0,66$ no ano de 2000), ficando atrás somente de outros três estados: Piauí, Alagoas e Maranhão${ }^{1}$.

As desigualdades regionais também estão expressas nos indicadores nutricionais, como destacado na Pesquisa Nacional Sobre Saúde e Nutrição (PNSN), de 1989, que mostrou maior pro- porção de desnutrição crônica entre crianças menores de cinco anos nas regiões Norte $(23,0 \%)$ e Nordeste $(27,3 \%)$ comparado com as regiões Sul $(8,7 \%)$, Sudeste $(8,1 \%)$ e Centro Oeste $(8,25)^{2}$.

Pesquisa realizada pelo $\mathrm{M}$ inistério do Desenvolvimento Social e Combate à Fome, no ano de 2005, identificou melhoras significativas nas prevalências dos déficits antropométricos. $A$ região do semi-árido nordestino apresentou prevalências em torno de $6,6 \%$ de déficit de altura para idade e 5,6\% de déficit de peso para idade 3 . $\mathrm{Na}$ Paraíba as prevalências observadas para estes mesmos índices foram de 3,7\% e 5,9\% respectivamente, mostrando uma situação ainda mais favorável com relação à desnutrição pregressa ${ }^{4}$. 
De acordo com a definição construída na II Conferência Nacional de Segurança Alimentar e Nutricional, realizada em Olinda no ano de 2004, a segurança alimentar é: "a realização do direito de todos ao acesso regular e permanente de alimentos de qualidade, em quantidade suficiente, sem comprometer 0 acesso a outras necessidades essenciais, tendo como base práticas alimentares promotoras de saúde, que respeitem a diversidade cultural e que sejam social, econômica e ambientalmente sustentáveis ${ }^{5}$ (p.1)".

O Direito Humano a uma Alimentação Adequada ${ }^{6}$ atualmente tem respaldo na Lei Orgânica de Segurança Alimentar e Nutricional $(\text { LOSAN })^{7}$, sancionada pelo Presidente da República em setembro de 2006, e apoio institucional na estratégia Fome Zero ${ }^{8}$ do Governo Federal, que busca assegurar este direito às pessoas com dificuldade de acesso aos alimentos, pela expansão da produção e do consumo de alimentos saudáveis, geração de ocupação e de renda, e pela melhoria da escolarização, das condições de saúde e do acesso ao abastecimento de água.

Pelas características da definição de Segurança A limentar e Nutricional, aspectos subjetivos como a própria percepção da fome, a escolha dos alimentos e até 0 medo de vir a passar fome ${ }^{9}$ são componentes deste conceito.

No ano de 2003 foi realizado um estudo multicêntrico ${ }^{10}$ que adaptou e validou para a realidade sócio-cultural do Brasil uma escala de medida direta da segurança e insegurança Alimentar desenvolvida nos Estados Unidos da América no início dos anos $90^{11}$ e utilizada no censo daquele país desde $1995^{12}$.

$O$ desenvolvimento da Escala Brasileira de Insegurança Alimentar (EBIA) possibilitou a realização de diversas pesquisas locais e de uma pesquisa nacional, a Pesquisa Nacional por Amostras Domiciliares (PNAD), conduzida pelo Instituto Brasileiro de Geografia e Estatística (IBGE) no ano de 2004. Esse trabalho identificou que 34,8\% da população brasileira sofrem com algum grau de insegurança alimentar, representando um importante problema de saúde pública. A prevalência de insegurança alimentar, entretanto, apresenta variações de acordo com as regiões do País, com destaque para as piores condições no Norte e Nordeste do País, o que confirma as desigualdades medidas por outros indicadores. Enquanto no Brasil, a insegurança alimentar grave comprometia o bem estar de 6,5\% dasfamílias, esta prevalência foi de $10,9 \%$ no Norte e $12,4 \%$ no Nordeste, atingindo $15,0 \%$ das famílias no Estado da Paraíba ${ }^{13}$.

Conhecer a prevalência de insegurança alimentar (IA) é de grande importância para a avaliação das condições de vida e, conseqüentemente, para o planejamento de políticas públicas de combate à fome. Neste sentido foi realizado um estudo de base populacional em municípios do interior do estado da Paraíba, com a finalidade de conhecer melhor a situação do Estado, quantificar a prevalência de insegurança alimentar familiar e relacioná-la com o perfil social, demográfico e econômico das famílias desses municípios. Esta iniciativa se faz necessária para a obtenção de informações inéditas, que podem ser utilizadas como indicadores para comparações regionais, nacionais e internacionais. $A$ investigação desagregada em municípios do interior do estado permite conhecer características locais que podem não ser percebidas em unidades de análise regionais ou estaduais. Estas características, comuns à grande maioria dos municípios do semi-árido nordestino, mostram as conseqüências das desigualdades regionais e possibilitam dimensionar o contingente populacional sob risco de ocorrência de problemas nutricionais.

\section{M ÉTO D O S}

Foi realizado um estudo seccional de base populacional em 14 municípios do interior do Estado da Paraíba durante os meses de maio a outubro de 2005. Os municípios escolhidos para participar do estudo foram aqueles selecionados pelo Governo Federal em 2003 para iniciar o programa Fome Zero, identificados, segundo o antigo Ministério Extraordinário de Segurança 
Alimentar (M ESA), hoje M inistério do Desenvolvimento Social e Combate à Fome, como os mais carentes do interior do Estado da Paraíba. Desses municípios, um se localiza na Zona da Mata, 10 na região Agreste, dois na Borborema e um no Sertão.

A amostra foi definida por amostragem aleatória estratificada, na qual cada município foi dividido por área urbana e rural e os tamanhos das amostras nos estratos foram calculados adotando-se partilha proporcional.

Com base nos dados populacionais do censo demográfico de $2000^{14}$ foram calculados os tamanhos das amostras para cada município. Utilizou-se erro amostral máximo de $5 \%$ sob nível de confiança de $95 \%$. A estimativa esperada da prevalência de segurança alimentar foi de $50 \%$ para maximizar o tamanho da amostra. Segundo estes parâmetros o tamanho calculado da amostra total foi de 4644 domicílios.

A partir dos mapas cartográficos de cada município, fornecidos pelo IBGE, foram sorteados aleatoriamente quarteirões na área urbana e aglomerados residenciais na área rural. 0 número de quarteirões necessários em cada município foi estimado de acordo com a densidade de famílias dos respectivos setores censitários. Na área rural procurou-se visitar o maior número possível de aglomerados residenciais.

Para o caso de não localização do número esperado de famílias nos quarteirões selecionados, os entrevistadores completavam o tamanho da amostra realizando visitas em um ou dois quarteirões extras, sorteados também aleatoriamente, no momento do planejamento do trabalho de campo, evitando a diminuição do tamanho da amostra e, conseqüentemente, a introdução de vieses na pesquisa.

Foram realizadas entrevistas domiciliares nas quais os chefes das famílias foram convidados a participar da pesquisa e, em caso de aceitação, assinaram um termo de consentimento livre e esclarecido.

As entrevistas foram conduzidas por entrevistadores selecionados em cada município, devendo atender os requisitos de ter, pelo menos, ensino médio completo, ter disponibilidade de tempo e não ser Agente Comunitário de Saúde ou desenvolver alguma atividade institucional ligada à Secretaria de Saúde. Todos os entrevistadores foram submetidos a um treinamento de 16 horas, sendo considerados aptos após a realização, de forma satisfatória, de um teste de aplicação do questionário da pesquisa.

Um questionário pré-testado foi utilizado para coleta de dados, sendo composto por:

- Caracterização da família: identificação do chefe da família, total de moradores por domicílio segundo idade, sexo e escolaridade e ocupações de cada morador;

- Dados sócio-demográficos: local de residência, tipo de construção da moradia, acesso a serviços públicos de água, esgoto e coleta de lixo;

- Escala Brasileira de Insegurança Alimentar (EBIA): 15 perguntas, destinadas a famílias com algum morador menor de 18 anos ou 9 perguntas para famílias compostas somente por adultos, com graus de gravidade crescente, indo desde a preocupação com a falta de alimento no domicilio, até a situação de algum morador haver passado um dia inteiro sem comer nos últimos três meses;

- Afirmativas sobre problemas relacionados à insegurança alimentar;

- Informações de rendimentos familiares: renda de cada morador e a participação da família em programas de doação de alimentos ou complementação de renda, neste caso com a quantificação do valor monetário recebido.

A classificação das famílias segundo segurança alimentar e graus de insegurança foi feita conforme a metodologia da EBIA ${ }^{9}$. Quando 0 entrevistado responde negativamente a todas as questões da escala, a família é classificada em situação de segurança alimentar, até 5 respostas positivas, insegurança alimentar leve, o que representa restrição na qualidade dos alimentos consumidos; de 6 a 10 respostas positivas, 
insegurança alimentar moderada, representando restrição na quantidade de alimentos e de 11 a 15 respostas positivas, insegurança alimentar grave, que representa um estado no qual a família convive com a situação real de fome, na qual os adultos e/ou as crianças residentes nesses domicílios deixam de realizar refeições ou mesmo ficam até um dia inteiro sem comida. Para famílias somente com adultos, a escala classifica em segurança alimentar quando todas as respostas são respondidas negativamente, em insegurança alimentar leve: de 1 a 3 respostas positivas, insegurança alimentar moderada: de 4 a 6 respostas positivas, insegurança alimentar grave: de 7 a 9 respostas positivas.

A consistência interna da escala foi medida com a estatística alfa de Cronbach, utilizando como valores aceitáveis resultados acima de 0,85. As medidas de associação entre a variável dependente, segurança alimentar, e as demais variáveis do estudo foram feitas utilizando-se o teste qui-quadrado com a probabilidade igual ou menor que 5\% para negação da hipótese de não associação. As variáveis que apresentaram associação significante foram incluídas em um modelo de regressão logística multivariada, utilizando o método de adição progressiva das variáveis, tendo como critério de inclusão o nível de significância da estatística Wald menor ou igual a $5 \%$ e de exclusão maior ou igual a $10 \%$. Foram calculados os valores de Odds Ratio e os respectivos intervalos de confiança de $95 \%$ das variáveis que permaneceram na equação do modelo multivariado.

Todos os protocolos referentes a cada família foram guardados em arquivo fechado com garantia de confidencialidade e as informações transcritas para meio digital, usando o utilitário Access do pacote Office for W indows. As análises foram desenvolvidas com o auxílio da plataforma estatística Statistical Package for Social Sciences (SPSS) for W indows, versão 8.

O projeto foi aprovado pelo Comitê de Ética em Pesquisa do Centro de Ciências da Saúde da Universidade Federal da Paraíba, na sua reunião ordinária no dia 17 de novembro de 2004, de acordo com a resolução 196/96 do Conselho Nacional de Saúde (MS).

\section{RES U LTA D O S}

Foram realizadas 4533 entrevistas domiciliares nos municípios selecionados. A média de integrantes por família foi de 3,98 (desvio-padrão de 1,86$)$, sendo envolvidas na pesquisa o total de 18088 pessoas, das quais 47,9\% eram do sexo masculino.

A avaliação da situação de segurança alimentar e dos diferentes graus de insegurança dos 14 municípios e do consolidado total é apresentada na Tabela 1. Os municípios estão classificados em ordem decrescente com relação à prevalência de segurança alimentar observada. De acordo com este critério de classificação, o município de Umbuzeiro foi o que apresentou a melhor situação de segurança alimentar e o de São José dos Ramos, a mais precária. Comparativamente, situações menos graves também foram observadas nos municípios de Picuí, Bananeiras, Cacimba de Dentro e Boqueirão, onde as prevalências de insegurança grave foram menores que $10 \%$ e as de segurança alimentar, próximas ou acima de $50 \%$.

Do total das entrevistas, 2090 foram realizadas na zona rural e 2443 na zona urbana. Situação de menor prevalência de segurança alimentar foi observada na primeira $(p<0,01)$, (44,5\% versus (vs) $50,1 \%$ ) e maior de insegurança alimentar grave $(14,0 \%$ vs $9,0 \%)$.

Os principais motivos relacionados à situação de insegurança alimentar, referidos pelos 2355 entrevistados classificados em insegurança leve, moderada ou grave, de acordo com uma lista pré-definida, são mostrados no Tabela 2.

Os motivos referidos foram diferenciados, quando divididos nas áreas de moradia urbana e rural. A falta de dinheiro para a aquisição de alimentos e a falta de produção agrícola foram os motivos mais referidos, sendo ambos mais freqüentes na área rural. Estar endividado ou sem 
crédito e a limitação do acesso aos mercados de alimentos também tiveram diferença estatística significante, com maior freqüência na área rural. Somente o fator "dieta especial" foi mais freqüente na área urbana, apesar de ser o segundo menos referido.

As famílias relacionaram com maior freqüência a falta de combustível, em terceiro lugar na lista, com a insegurança alimentar, comparado com a falta de água, que foi o menos referido. Ambos os fatores não apresentaram diferença significante entre as áreas urbana e rural.

As condições de moradia, o acesso à rede de serviços e a renda familiar per capita estiveram fortemente associadas com a situação de insegurança alimentar (todas as variáveis apresentaram $p<0,01$, segundo o teste qui-quadrado) e foram incluídas em um modelo de regressão logística multivariado, ajustado pelo local de moradia (urbano e rural), tendo segurança alimentar definida como variável dependente dicotômica (sim ou não).

Os resultados da regressão logística apresentam os valores de Odds Ratio e intervalo de confiança de $95 \%$ das variáveis que permaneceram no modelo final, como é apresentado na Tabela 3.

Tabela 1. Prevalência da condição de segurança alimentar e dos graus de insegurança alimentar. Paraíba, 2005.

\begin{tabular}{lccccr}
\hline \multirow{2}{*}{ Municípios* } & \multirow{2}{*}{ Entrevistados (n) } & Segurança alimentar $(\%)$ & \multicolumn{3}{c}{ Insegurança (\%) } \\
\cline { 4 - 6 } & 251 & 64,1 & Leve & Moderada & Grave \\
\hline Umbuzeiro & 361 & 62,7 & 19,1 & 10,0 & 6,8 \\
Bananeiras & 349 & 59,3 & 17,5 & 11,4 & 8,5 \\
Aroeiras & 316 & 57,5 & 16,0 & 14,3 & 10,3 \\
Picuí & 325 & 52,9 & 23,0 & 14,1 & 5,4 \\
Nova Floresta & 342 & 50,0 & 20,0 & 15,1 & 12,0 \\
Cacimba de Dentro & 349 & 48,6 & 26,2 & 16,4 & 7,4 \\
Boqueirão & 269 & 48,5 & 31,2 & 13,0 & 7,2 \\
Bernadino Batista & 361 & 45,4 & 20,1 & 20,1 & 11,2 \\
Esperança & 305 & 38,5 & 20,8 & 19,9 & 13,9 \\
Areial & 369 & 38,2 & 31,1 & 21,0 & 9,4 \\
Queimadas & 317 & 37,3 & 28,5 & 18,2 & 15,2 \\
Itabaiana & 335 & 32,2 & 26,6 & 23,7 & 12,3 \\
Araruna & 284 & 30,2 & 23,9 & 28,1 & 15,8 \\
São José dos Ramos & 433 & 47,5 & 25,3 & 21,7 & 22,8 \\
\hline Total & & & 23,6 & 17,6 & 11,3 \\
\hline
\end{tabular}

* Alfa de Cronbach entre 0,91 e 0,95 .

Tabela 2. Motivos identificados relacionados à condição de insegurança alimentar em função da área de moradia. Paraíba, 2005.

\begin{tabular}{lcccc}
\hline Motivos & Entrevistados $(\mathrm{n})$ & Urbano (\%) & Rural (\%) & Valor de $\mathrm{p}^{*}$ \\
\hline Faltou dinheiro para comida & 1502 & 45,3 & 54,7 & $<\mathbf{0 , 0 1}$ \\
Faltou produção de alimentos suficiente & 1250 & 46,6 & 53,4 & $<0, \mathbf{1}$ \\
Faltaram gás, lenha ou álcool para cozinhar & 944 & 51,8 & 48,2 & 0,07 \\
Faltou variedade da sua preferência & 804 & 51,4 & 48,6 & 0,21 \\
Estamos endividados/sem crédito & 787 & 46,4 & 53,6 & $<\mathbf{0 , 0 1}$ \\
É muito difícil chegar até o mercado & 685 & 32,6 & 67,4 & $<\mathbf{0 , 0 1}$ \\
Problemas de saúde & 532 & 48,7 & 51,3 & 0,29 \\
Faltou tempo para fazer compras ou cozinhar & 388 & 49,0 & 44,0 & 0,35 \\
Estamos em dieta especial & 331 & 55,9 & 49,1 & $\mathbf{0 , 0 1}$ \\
Faltou água para cozinhar & 279 & 50,9 & 0,40
\end{tabular}

* teste qui-quadrado; $\mathrm{p}$ em negrito: significância estatística. 
Tabela 3. Distribuição das famílias segundo a situação de segurança, os graus de insegurança alimentar e as condições de moradia e renda. Paraíba, 2005 ( $n=4$ 533).

\begin{tabular}{|c|c|c|c|c|c|c|c|}
\hline \multirow{2}{*}{ Variáveis do estudo } & \multirow{2}{*}{$\begin{array}{l}\text { Entrevistados } \\
\text { (n) }\end{array}$} & \multirow{2}{*}{$\begin{array}{c}\text { Segurança alimentar } \\
(\%)\end{array}$} & \multicolumn{3}{|c|}{ Insegurança (\%) } & \multirow{2}{*}{ OR } & \multirow{2}{*}{ (IC 95\%) } \\
\hline & & & Leve & Moderada & Grave & & \\
\hline \multicolumn{8}{|l|}{ Construção da moradia } \\
\hline Alvenaria acabada & 3617 & 51,9 & 23,6 & 15,6 & 8,9 & & 1 \\
\hline Outro tipo & 860 & 29,3 & 22,9 & 26,4 & 21,4 & 1,98 & $(1,64-2,38)$ \\
\hline \multicolumn{8}{|l|}{ Serviço de água* } \\
\hline Rede pública & 2031 & 51,7 & 24,3 & 15,3 & 8,7 & & \\
\hline Outro sistema & 2436 & 42,5 & 22,6 & 20,4 & 14,5 & & \\
\hline \multicolumn{8}{|l|}{ Disponibilidade de água } \\
\hline Tem água no domicílio & 3394 & 51,9 & 23,7 & 15,3 & 9,0 & & 1 \\
\hline Busca água fora & 1032 & 33,5 & 22,7 & 24,9 & 18,9 & 1,38 & $(1,15-1,65)$ \\
\hline \multicolumn{8}{|l|}{ Esgotamento sanitário* } \\
\hline Rede pública & 1233 & 54,3 & 25,5 & 13,3 & 6,9 & & \\
\hline Outro & 3235 & 44,8 & 22,8 & 19,4 & 13,0 & & \\
\hline \multicolumn{8}{|l|}{ Coleta de lixo* } \\
\hline Pública & 2653 & 50,3 & 24,7 & 15,5 & 9,5 & & \\
\hline Outra & 1811 & 43,0 & 22,1 & 20,9 & 14,0 & & \\
\hline \multicolumn{8}{|l|}{ Renda per capita } \\
\hline mais de 300 reais & 230 & 85,2 & 10,4 & 3,5 & 0,9 & & 1 \\
\hline de 200 a 300 reais & 594 & 72,4 & 18,0 & 6,4 & 3,2 & 2,18 & $(1,45-3,28)$ \\
\hline de 100 a 200 reais & 1087 & 57,3 & 23,6 & 13,7 & 5,4 & 4,19 & $(2,84-6,18)$ \\
\hline de 50 a 100 reais & 1154 & 42,0 & 26,9 & 21,0 & 10,1 & 7,72 & $(5,24-11,37)$ \\
\hline de 25 a 50 reais & 604 & 24,2 & 24,8 & 27,3 & 23,7 & 16,55 & $(10,89-25,16)$ \\
\hline até 25 reais & 243 & 21,8 & 23,5 & 27,6 & 27,2 & 19,10 & $(11,75-31,04)$ \\
\hline
\end{tabular}

*Excluído do modelo; OR: Odds Ratio; IC: intervalo de confiança.

A precariedade das condições de moradia esteve fortemente associada à IA das famílias, sendo que não morar em casa de alvenaria aumentou em quase duas vezes a chance de insegurança alimentar. Ter de buscar água fora do domicílio, por sua vez, aumenta 1,38 vezes a mesma chance.

0 rendimento mensal médio per capita esteve inversamente associado à situação de insegurança alimentar, ou seja, quanto menor o rendimento, maior a chance de insegurança alimentar. Esta associação é confirmada pelo comportamento dose/resposta da relação entre estas variáveis, pois se observa o progressivo aumento da chance de insegurança alimentar à medida que diminui a renda, chegando a ser quase 20 vezes maior para as famílias de rendimento até $\mathrm{R} \$ 25,00$ mensais per capita (aproximadamente
US $\$ 11,00$ ou 1/12 salário-mínimo), comparado com famílias com renda superior a $\mathrm{R} \$ 300,00$ mensais per capita (aproximadamente US\$130,00 ou 1,0 salário-mínimo).

Quando se perguntou às famílias sobre sua participação em programas de apoio social, 15,9\% delas responderam que "recebem algum tipo de doação de alimentos" e 35,3\% que "participam dos programas de complementação de renda". Análises estratificadas por situação de segurança alimentar e pelos diferentes graus de insegurança alimentar permitiram observar que à medida que aumentava o grau de insegurança alimentar, também aumentava a proporção de famílias que recebiam algum tipo de ajuda, sendo que, mais da metade das famílias em insegurança alimentar grave estavam incluídas em algum programa oficial de transferência de renda (Figura 1). 


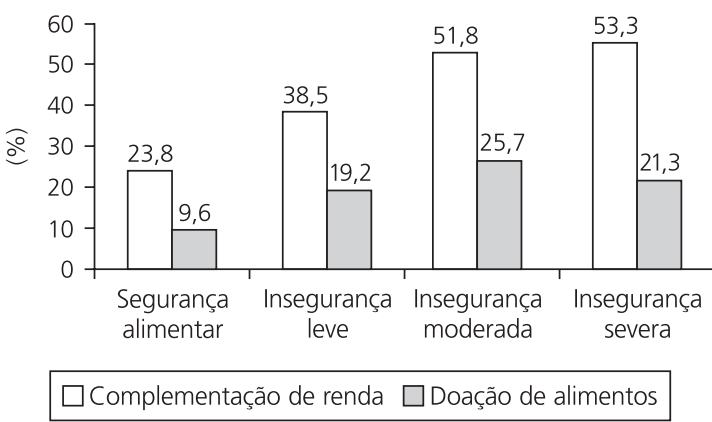

Figura 1. Porcentagem de famílias que recebem doação de alimentos ou que estão incluídas em algum programa de complementação de renda, segundo a condição de segurança alimentar e os graus de insegurança alimentar. Paraíba, 2005 ( $n=4$ 533).

Ao considerar somente as famílias com o menor rendimento per capita (até $R \$ 25,00$ ), condição que concentra a maior proporção de beneficiários, comparou-se a prevalência de segurança e insegurança alimentar entre as famílias que participam de programas de alimentação - como merenda escolar, cestas básicas etc., - com as famílias que não participam, assim como entre as famílias que recebem algum benefício em dinheiro - como bolsa escola, vale gás, bolsa alimentação, bolsa família - com as demais famílias (Tabela 4).

Controlado pela renda, observou-se prevalência menor de insegurança alimentar grave entre as famílias inscritas nos programas, seja os de doação de alimentos (redução de 7,1\%), ou aqueles de complementação de renda (redução de 4,8\% ), embora a associação entre estas duas variáveis não seja estatisticamente significante pelo teste qui-quadrado.
D ISCUSSÃ O

Este trabalho apresentou o resultado de um estudo de base populacional, no qual foram incluídos intencionalmente 14 municípios do estado da Paraíba. Não é possível afirmar que estes resultados expressam a situação global do estado da Paraíba, uma vez que o plano amostral da pesquisa não teve este objetivo. A diferença entre o número de domicílios planejados na amostra e aqueles efetivamente visitados foi menor que 2,4\%, o que assegura a não ocorrência de vieses por perda amostral. 0 critério de aleatoriedade das famílias entrevistadas foi respeitado, de acordo com a alocação dos domićlios nos quarteirões selecionados por sorteio em cada município. Portanto, os dados são representativos de cada município, como também do conjunto deles.

As entrevistas foram realizadas por entrevistadores locais, o que diminuiu muito os custos do trabalho de campo, pois dispensaram gastos com deslocamento, diárias e manutenção de uma equipe nos diferentes municípios. Optou-se por não trabalhar com Agentes Comunitários de Saúde, para evitar tendências nas respostas dos entrevistados, tanto pela expectativa de obtenção de algum benefício, quanto devido à possibilidade de vínculos anteriores existentes com as famílias.

Apesar do grande número de entrevistadores envolvidos, os resultados de cada município, bem com o consolidado dos 14 municípios mostraram-se consistentes, com validade psicométrica alta, medida pela estatística alfa de Cronbach. Este resultado mostra a possibilidade de aplicação desta metodologia em diferentes

Tabela 4. Distribuição das famílias com renda per capita até $R \$ 25,00$, segundo a situação de segurança e os graus de insegurança alimentar e a participação em Programas de Complementação de Renda e de Doação de Alimentos. Paraíba, 2005.

\begin{tabular}{|c|c|c|c|c|c|}
\hline \multirow{2}{*}{ Condição } & \multicolumn{2}{|c|}{ Complementação de renda (\%) } & \multicolumn{2}{|c|}{ Doação de alimentos (\%) } & \multirow{2}{*}{ Total $(\%) \quad(n=500)$} \\
\hline & $\operatorname{Sim}(n=363)$ & Não $(n=137)$ & $\operatorname{Sim}(n=161)$ & Não $(n=339)$ & \\
\hline Segurança alimentar & 17,6 & 22,6 & 17,4 & 19,8 & 19,0 \\
\hline Insegurança leve & 23,4 & 21,9 & 22,4 & 23,3 & 23,0 \\
\hline Insegurança moderada & 33,1 & 24,8 & 37,9 & 27,4 & 30,8 \\
\hline Insegurança severa & 25,9 & 30,7 & 22,4 & 29,5 & 27,2 \\
\hline Total & 100,0 & 100,0 & 100,0 & 100,0 & 100,0 \\
\hline
\end{tabular}


municípios, de forma descentralizada, sempre que o requisito de treinamento adequado dos entrevistadores seja atendido.

A Pesquisa Nacional por Amostras de Domicílios, realizada pelo IBGE no ano de 2004, investigou a situação de insegurança alimentar nos domicílios em todo o território nacional, com possibilidade de desagregação em Grandes Regiões e Unidades da Federação. Os resultados desta pesquisa mostraram que no Brasil 65,2\% da população encontra-se em segurança alimentar, sendo que essa prevalência é um pouco menor nas áreas rurais $(56,5 \%)$. A companhando as diferenças regionais, a situação de segurança alimentar é menos freqüente nos estados das regiões Norte $(53,6 \%)$ e Nordeste $(46,6 \%)$, quando comparada com as outras regiões do País. Na Paraíba, a PNAD identificou prevalência de segurança alimentar de $46,7 \%$, e de insegurança alimentar leve de 17,4\%, moderada de 20,8\% e grave de $15,1 \%{ }^{13}$. Esses resultados são próximos aos valores obtidos no consolidado dos 14 municípios, demonstrando consistência e reprodutibilidade da metodologia utilizada.

Situação mais desfavorável com relação à segurança alimentar, segundo este trabalho, foi observada na zona rural. Este resultado também concorda com o observado na PNAD para a região Nordeste e para o estado da Paraíba. As diferenças observadas entre os municípios devem ser investigadas considerando as características locais de produção e organização social, o que não foi contemplado no presente trabalho.

A medida de Segurança Alimentar esteve diretamente relacionada com as condições socioeconômicas das famílias entrevistadas, sendo observado neste trabalho que a baixa renda, 0 tipo de construção da moradia e a disponibilidade de água nos domicílios aumentaram a chance de as famílias estarem em situação de insegurança alimentar. Entretanto, a medida de segurança alimentar ou os diferentes graus de insegurança alimentar não são apropriados para a realização de uma avaliação do nível socioeconômico, pois, mesmo em famílias de maior poder aquisitivo ou de melhores condições de moradia observam-se situações desfavoráveis com relação à segurança alimentar, especialmente porque este é um conceito mais amplo, conforme sua definição.

Anteriormente à realização da pesquisa do IBGE, dois outros inquéritos de base populacional já haviam sido realizados, no município de Campinas $^{15}$ e no Distrito Federal16. Os resultados mostraram, nos dois casos, prevalências de segurança alimentar entre as famílias em torno de 40,0\%, valores abaixo do observado pelo IBGE, e também menores que o resultado da Paraíba, segundo este trabalho. Porém, os valores de insegurança alimentar grave para Campinas e Brasília foram, respectivamente, $6,6 \%$ e $7,6 \%$ os quais, apesar de demonstrarem a importância da gravidade da situação diagnosticada, confirmam as desigualdades regionais, que afetam com maior severidade as áreas menos desenvolvidas, como é o caso dos municípios do estado da Paraíba.

Vale observar que, em ambas as pesquisas, apesar da insegurança alimentar grave, que diagnostica situação de fome entre os integrantes da família, não haver sido observada em famílias com rendimentos acima de um salário-mínimo per capita, mesmo em famílias com renda familiar per capita de até três salários-mínimos, ainda foram observadas situações de insegurança alimentar leve e, em menor freqüência, moderada. Resultado semelhante foi observado na Paraíba, quando famílias com renda per capita acima de $R \$ 300,00$, equivalente a um salário-mínimo na época da coleta de dados, praticamente não reportaram situações de insegurança alimentar grave, apesar de ainda serem classificadas nos outros graus menos severos de insegurança. Este resultado é especialmente importante, pois possibilita identificar um limite mínimo para a renda que permitiria às famílias, mesmo em distintas regiões, viver protegido da experiência da fome.

Pesquisa realizada com famílias com idosos em Campinas (SP) observou que, mesmo em famílias pertencentes ao estrato socioeconômico alto, a prevalência de insegurança alimentar leve chegava a $25,0 \%$. Nos estratos de nível médio 
esta prevalência subia para $54,6 \%$, sendo $34,0 \%$ leve, $13,4 \%$ moderada e $7,2 \%$ grave. 0 fator considerado nesta pesquisa para a divisão entre os grupos foi a proporção de escolaridade superior ${ }^{17}$. Este resultado reafirma que a insegurança alimentar é um fenômeno mais complexo que aquele medido somente por indicadores socioeconômicos, embora esteja muito relacionada a eles.

No presente estudo não foi possível classificar as famílias em estratos com mais de um salário-mínimo familiar per capita mensal, devido ao número muito pequeno de famílias nestas condições. Esta limitação impede comparações das prevalências de segurança alimentar entre grupos de maior renda com os estudos de Campinas ou Brasília.

0 instrumento utilizado nesta pesquisa para a avaliação da segurança alimentar, que foi adaptado e validado para a realidade brasileira, também foi validado e utilizado em diferentes países. Na América Latina, nos últimos cinco anos, inquéritos foram realizados na Colômbia, no M éxico, Bolívia, na Venezuela e em Trinidad e Tobago ${ }^{18}$, assim como em países de outros continentes, como Burkina Faso, na África, e Filipinas, na Ásia ${ }^{19}$. Os resultados encontrados nessas pesquisas mostram como os diferentes meios de produção e as diferentes culturas influenciam na situação de segurança alimentar e que as prevalências observadas de insegurança alimentar também são altas, variando desde 35,5\% nas Filipinas, 70,3\% na Bolívia e mais de $90,0 \%$ no estado de Jalisco (M éxico) ${ }^{18,19}$.

No Brasil, a Pesquisa de Orçamentos Familiares de 2002-2003 fez uma avaliação subjetiva das condições de vida, em que foi perguntado sobre a dificuldade de ajustar o rendimento familiar mensal para co brir todos os gastos da família durante o mês, bem como sobre a qualidade e a suficiência dos alimentos consumidos pelas famílias. Na Paraíba, 27,01\% das famílias referiram que, raramente, comem o tipo de alimento preferido e, ainda, 25,77\% referiram que a quantidade de alimentos mensais normalmente é insuficiente ${ }^{20}$. Estas medidas mostram uma correspondência muito forte com os achados de campo obtidos com a utilização da EBIA na Paraíba, que encontrou $11,30 \%$ de insegurança grave e $17,60 \%$ de insegurança moderada, totalizando $28,90 \%$ das famílias com restrição na quantidade de alimentos consumidos. Também, 23,60\% das famílias apresentaram insegurança alimentar leve, que se caracteriza pela preocupação com a suficiência dos alimentos no domicílio e com a substituição da dieta, comprometendo a qualidade da mesma.

As alterações indevidas das dietas das populações têm afetado de modo negativo o estado nutricional de grande contingente de pessoas. A subnutrição e a obesidade passaram a ser problemas nutricionais concomitantes nos países em desenvolvimento e o segundo pode ser explicado, para populações de baixo poder aquisitivo, pela escolha de alimentos com alta densidade energética e baixo custo ${ }^{21}$, bem como por características culturais e simbólicas relacionadas à alimentação e à saúde ${ }^{22}$. Porém, mesmo situações adversas e opostas constituem elementos inerentes da definição de insegurança alimentar, uma vez que não somente a falta, mas também o respeito aos hábitos alimentares são premissas do conceito definido na II Conferencia Nacional de Segurança Alimentar.

Os resultados dos estudos utilizando a EBIA apresentam muita consistência entre si e guardam correspondência muito forte com os achados da Pesquisa de Orçamento Familiar (POF), como descrito anteriormente. Entretanto as prevalências de insegurança alimentar não correspondem, em ordem de grandeza, aos recentes resultados de avaliação nutricional de crianças menores de cinco anos, realizado no semi-árido brasileiro. As prevalências de déficits de peso ou altura para idade no estado da Paraíba, que foram 5,9\% e 3,7\%, respectivamente ${ }^{4}$, são muito menores que as prevalências de insegurança alimentar, mesmo considerando somente a insegurança alimentar grave $(11,3 \%$ ). Somado a isso, devem-se considerar os casos observados de excesso de peso para altura, cuja freqüência na Paraíba foi de $6,2 \%{ }^{23}$, reve- 
lando outro problema nutricional emergente. Por estes motivos entende-se que estes indicadores antropométricos para diagnosticar o estado nutricional e a EBIA para avaliar a segurança alimentar, medem apropriadamente fenômenos diferentes.

Sobre os programas sociais, tanto de doações de alimentos como de complementação de renda, os resultados apresentados neste trabaIho, com relação à redução da insegurança alimentar grave nas famílias classificadas no estrato de menor rendimento mensal, apontam para um possível resultado positivo desses programas na redução da situação de fome entre famílias dessa classe de rendimento. Estes dados ainda não são suficientes para um diagnóstico de avaliação do impacto desses programas, pela inexistência de parâmetros de comparação para a situação observada. À parte esta limitação, foi observado que as famílias com menor rendimento e classificadas em graus mais graves de insegurança alimentar, tiveram maior participação nestes programas, mostrando um direcionamento adequado dos recursos envolvidos. Resultado positivo de direcionamento adequado dos recursos de programas sociais também foi observado em um estudo desenvolvido em Piracicaba (SP), onde 73,9\% das famílias beneficiadas com programas de complementação de renda pertenciam ao estrato de renda igual ou inferior a 0,5 salário-mínimo per capita ${ }^{24}$. Os programas de complementação de renda apresentam também outros aspectos positivos, que não foram mensurados no presente trabalho, mas devem ser destacados, como o fortalecimento da economia local, os baixos custos operacionais e a possibilidade de autonomia dos usuários na utilização dos recursos ${ }^{25}$.

\section{CONSIDERAÇÕ ES FINA IS}

Vários estudos estão sendo realizados em diversas regiões do Brasil utilizando a Escala Brasileira de Insegurança Alimentar, assim como em outros países, o que possibilita aumentar a compreensão sobre este fenômeno e, especial- mente, encontrar formas de enfrentamento por meio de políticas públicas promotoras do Direito Humano à Alimentação Adequada e da garantia de Segurança Alimentar e Nutricional, como preconiza o relatório final da III Conferência Nacional de Segurança Alimentar e Nutricional.

Os resultados do presente estudo mostram informações novas e importantes sobre a situação de segurança alimentar de municípios do estado da Paraíba, servindo de referência para a região e para futuros estudos avaliativos. Estudar as diferenças de prevalência de segurança alimentar e dos diferentes graus de insegurança alimentar entre os 14 municípios deste estudo irá fornecer ferramentas importantes para a elaboração, 0 monitoramento e a avaliação de políticas sociais locais e deverá ser as próximas etapas desta pesquisa.

Conhecer a prevalência da insegurança alimentar nos municípios do interior do estado da Paraíba e os fatores identificados pela população que impactam negativamente na garantia de uma alimentação saudável e adequada, de maneira continua e sustentável, assim como encontrar as características sócio-demográficas diretamente associadas à condição de insegurança alimentar são as principais contribuições do presente trabalho.

A metodologia utilizada demonstrou ter grande potencial para a realização de diagnósticos locais e regionais com rapidez e baixo custo, mensurando diretamente a situação de segurança e insegurança alimentar familiar, sendo uma importante ferramenta que demonstrou ser capaz de ser utilizada para o monitoramento e a avaliação de políticas públicas, como os programas de doação de alimentos ou complementação de renda, inseridos na estratégia Fome Zero do Governo Federal.

\section{COLABORADORES}

R.P.T. VIANNA realizou o planejamento, a coordenação da coleta de dados, as análises e a redação do manuscrito. A.M. SEGALL-CORRÊA participou do desenvolvimento da metodologia, da interpretação dos dados e da revisão do manuscrito. 


\section{RE FERÊ N CIAS}

1. Programa das Nações Unidas para o Desenvolvimento. Atlas do desenvolvimento humano no Brasil. Brasília; 2003.

2. Instituto Nacional de Alimentação e Nutrição. Pesquisa nacional sobre saúde e nutrição. Resultados preliminares. Brasília: Ministério da Saúde; 1990.

3. Monteiro CA, Conde WL, Konno SC. Análise do inquérito chamada nutricional: 2005. Cad Estud Desenv Soc Debate. 2006; 4(junho):29-37.

4. Dantas M BP, Vianna RPT, Almeida ESG. A chamada nutricional na Paraíba: avaliação de crianças menores de cinco anos na região do semi-árido paraibano. Cad Estud Desenv Soc Debate. 2006; 4(junho):61-8.

5. Brasil. Conselho Nacional de Segurança Alimentar e Nutricional. II Conferência Nacional de Segurança Alimentar e Nutricional [documento final]. Olinda; 2004.

6. Valente FLS. Fome, desnutrição e cidadania: inclusão social e direitos humanos. Saúde e Soc. 2003; 12:51-60.

7. Brasil. Conselho Nacional de Segurança Alimentar e Nutricional. III Conferência Nacional de Segurança Alimentar e Nutricional [documento final]. Fortaleza; 2007.

8. Brasil. M inistério do Desenvolvimento Social. Fome zero: conceito. Brasília. 2007 [acesso 2007 nov]. Disponível em: <http://www.fomezero.gov.br/oque-e>.

9. Radimer KL, Olson CM, Greene JC, Campbell CC, Habicht JP. Understanding hunger and developing indicators to assess it in woman and children. J Nutr Educ. 1992; 24(Supl):36-44

10. Segall-Corrêa AM, Perez-Escamilla R, Sampaio M FA, Marin-León L, Panigassi G, Maranha LK, et al. Acompanhamento e avaliação da segurança alimentar de famílias brasileiras: validação de metodologia e de instrumento de coleta de informação: urbano/rural. Campinas: Universidade Estadual de Campinas; 2004 [acesso 2007 jul]. Disponível em: <http://www.opas.org.br/publicacl. cfm>.

11. Bickel G, Nord M, Price C, Hamilton W, Cook J. $M$ easuring food security in the United States: guide to measuring household food security. Washington (DC): USDA; 2000.

12. Radimer KL, Measurament of household food security in the USA and other industrialized countries. Public Health Nutr. 2002; 5(6A):859-64.
13. Instituto Brasileiro de Geografia e Estatística. Pesquisa Nacional por Amostra de Domicílios. Segurança alimentar: 2004. Rio de Janeiro; 2006.

14. Instituto Brasileiro de Geografia e Estatística. Censos demográficos e contagens populacionais: 2003. Rio de Janeiro [acesso 2007 jul]. Disponível em: <http://www. datasus.gov.br>.

15. Panigassi G. Inquérito populacional sobre a percepção da segurança alimentar intrafamiliar no município de Campinas, SP [tese]. Campinas: Unicamp; 2005.

16. Leão M. Segurança alimentar e risco de sobrepeso e obesidade em famílias de crianças menores de 6 anos [dissertação]. Brasília: UnB; 2005.

17. Marin-Leon L, Segall-Correa AM, Panigassi G, Maranha LK, Sampaio MFA, Perez-Escamilla R. A percepção de insegurança alimentar em famílias com idosos em Campinas, São Paulo, Brasil. Cad Saúde Pública. 2005; 21(5):1433-40.

18. Hackett M, Zubieta AC, Hernandez K, MelgarQuiñonez $\mathrm{H}$. Food insecurity and household food supplies in rural Ecuador. ALAN. 2007; 57(1):10-7.

19. Melgar-Quinonez $H R$, Zubieta AC, M knelly $B$, Nteziyaremye A, Gerardo MF, Dunford C. Household food insecurity and food expenditure in Bolivia, Burkina Faso, and the Philippines. J Nutr. 2006; 136(5):1431S-7S.

20. Instituto Brasileiro de Geografia e Estatística. Pesquisa de orçamentos familiares: 2002-2003. Resultados preliminares. Rio de Janeiro; 2004.

21. Caballero B. Subnutrição e obesidade em países em desenvolvimento. Cad Estud Desenv Soc Debate. 2005; 2(novembro):10-3.

22. Ferreira VA, Magalhães R. Obesidade e pobreza: 0 aparente paradoxo. Um estudo com mulheres da Favela da Rocinha, Rio de Janeiro, Brasil. Cad Saúde Pública. 2005; 21(6):1792-800.

23. Ferreira HS, Assunção ML, Florêncio TMMT, Lima MAA. Estado Nutricional de pré-escolares da região semi-árida do estado de Alagoas, 2005. Cad Estud Desenv Soc Debate. 2006; 4(F):37-42.

24. Silva MV, Sturion GL. Estado nutricional, acesso aos programas sociais e aquisição de alimentos. Saúde Rev. 2004; 6(13):53-61.

25. Burlandy L. Transferência condicionada de renda e segurança alimentar e nutricional. Ciênc Saúde Coletiva. 2007; 12(6):1441-51.

Recebido em: 6/3/2007

Versão final reapresentada em: 26/2/2008 Aprovado em: 29/2/2008 